\title{
TAME DIVISION ALGEBRAS OF PRIME PERIOD OVER FUNCTION FIELDS OF $p$-ADIC CURVES
}

\author{
ERIC BRUSSEL AND EDUARDO TENGAN
}

\begin{abstract}
Let $\mathrm{F}$ be a field finitely generated and of transcendence degree one over a $p$-adic field, and let $\ell \neq p$ be a prime. Results of Merkurjev and Saltman show that $\mathrm{H}^{2}\left(F, \mu_{\ell}\right)$ is generated by $\mathbb{Z} / \ell$-cyclic classes. We prove the " $\mathbb{Z} / \ell$-length" in $\mathrm{H}^{2}\left(F, \mu_{\ell}\right)$ equals the $\ell$-Brauer dimension, which Saltman showed to be two. It follows that all $F$-division algebras of period $\ell$ are crossed products, either cyclic (by Saltman's cyclicity result) or tensor products of two cyclic division algebras. Our result was originally proved by Suresh when $F$ contains the $\ell$-th roots of unity $\mu_{\ell}$.
\end{abstract}

\section{INTRODUCTION}

Let $F$ be a field and $n$ a number prime-to-char $(F)$. Suppose the cup product map $\mathrm{H}^{1}\left(F, \mu_{n}\right) \otimes_{\mathbb{Z}} \mathrm{H}^{1}(F, \mathbb{Z} / n) \rightarrow \mathrm{H}^{2}\left(F, \mu_{n}\right)={ }_{n} \operatorname{Br}(F)$ is surjective. This is the case when $F$ contains the $n$-th roots of unity or $n=3$, by Merkurjev-Suslin's theorem 7 , Theorem 16.1, Corollary 16.4], but unknown in general. Let the $n$-Brauer dimension ${ }_{n} \operatorname{Br} \cdot \operatorname{dim}(F)$ denote the smallest number $d$ such that every class in $\mathrm{H}^{2}\left(F, \mu_{n}\right)$ has index dividing $n^{d}$, and let the $\mathbb{Z} / n$-length ${ }_{n} \mathrm{~L}(F)$ denote the smallest number of $\mathbb{Z} / n$-cyclic classes needed to write any class in $\mathrm{H}^{2}\left(F, \mu_{n}\right)$. It is easy to see that ${ }_{n} \operatorname{Br} \cdot \operatorname{dim}(F) \leq{ }_{n} \mathrm{~L}(F)$, but even when the cup product map is surjective it is not known in general whether finite $n$-Brauer dimension implies finite $\mathbb{Z} / n$-length.

Suppose $F$ is finitely generated of transcendence degree one over the $p$-adic field $\mathbb{Q}_{p}$. Saltman showed ${ }_{n} \operatorname{Br} \cdot \operatorname{dim}(F)=2$ in [8, Theorem 3.4]. Recently Suresh showed that ${ }_{n} \mathrm{~L}(F)=2$ when $n=\ell$ is prime and $F$ contains $\mu_{\ell}$ ([12, Theorem 2.4]). The assumption on roots of unity excludes important cases such as the rational function field $F=\mathbb{Q}_{p}(T)$ (if $\ell \neq 2,3$ and $p \neq 1(\bmod \ell)$ ). But in this case the cup product map is surjective by Merkurjev's theorem [6, Theorem 2] and Saltman's cyclicity result for classes of prime index [11, Theorem 5.1], so $\mathrm{H}^{2}\left(F, \mu_{\ell}\right)$ is generated by $\mathbb{Z} / \ell$-cyclic classes. We show $\ell \mathrm{L}(F)=2$, hence all $F$-division algebras of period $\ell$ and index $\ell^{2}$ decompose into two cyclic $F$-division algebras of index $\ell$. It follows immediately that all $F$-division algebras of period $\ell$ are (abelian) crossed products. Noncrossed products of larger period exist by [2] and [3].

Our results rely on Saltman's degree- $\ell$ cyclicity result and hot point criterion [11, Corollary 5.2], and also on our lifting results from [3, which use the machinery of Grothendieck's existence theorem. We show an $F$-division algebra $\Delta$ of period $\ell$ and index $\ell^{2}$ is decomposable by constructing a tensor factor of degree $\ell$, lifting a class constructed over the generic points of the closed fiber of a 2-dimensional model

1991 Mathematics Subject Classification. 11G20, 11R58, 14E22, 16K50. 
$X / \mathbb{Z}_{p}$, as in [3]. We use Grunwald-Wang's theorem to construct the lifted class so that it "cancels" the hot points of $\Delta$, which implies it is part of a decomposition of $\Delta$ by the hot point criterion. The cyclicity result then shows the remaining factor is cyclic. Suresh's approach in [12] similarly cancels $\Delta$ 's hot points using a tensor factor, but his tensor factor is constructed as a symbol algebra, which requires $\mu_{\ell} \subset F$. We know of no way to get to the general case from that construction.

\section{Background And Conventions}

2.1. Brauer Group Conventions. In this paper an $F$-division algebra is a division ring that is central and finite-dimensional over $F$. If $D$ is an $F$-division algebra we write $[D]$ for the class of $D$ in the Brauer group $\operatorname{Br}(F)$, ind $(D)$ for the index or degree of $D$, and $\operatorname{per}(D)$ for the period of $D$. We say $D$ is a crossed product if it contain a maximal subfield that is Galois over $F$. See [1] for a discussion of crossed product and noncrossed product division algebras.

We write $\mathrm{H}^{2}\left(F, \mu_{n}\right)={ }_{n} \operatorname{Br}(F)$ for the $n$-torsion subgroup, where $n$ is primeto-char $(F)$ and $\mu_{n}$ is the group of $n$-th roots of unity. In the terminology of [1, Section 4], the $n$-Brauer dimension ${ }_{n} \operatorname{Br} \cdot \operatorname{dim}(F)$ is the smallest number $d$ such that every class in $\mathrm{H}^{2}\left(F, \mu_{n}\right)$ has index dividing $n^{d}$. We say $\mathrm{H}^{2}\left(F, \mu_{n}\right)$ is generated by $\mathbb{Z} / n$-cyclic classes if the cup product map $\mathrm{H}^{1}\left(F, \mu_{n}\right) \otimes_{\mathbb{Z}} \mathrm{H}^{1}(F, \mathbb{Z} / n) \rightarrow \mathrm{H}^{2}\left(F, \mu_{n}\right)$ is surjective, and a class is $\mathbb{Z} / n$-cyclic if it has the form $(f) \cdot \theta$ for some $(f) \in \mathrm{H}^{1}\left(F, \mu_{n}\right)$ and $\theta \in \mathrm{H}^{1}(F, \mathbb{Z} / n)$. When $\mathrm{H}^{2}\left(F, \mu_{n}\right)$ is generated by $\mathbb{Z} / n$-cyclic classes, we define the $\mathbb{Z} / n$-length ${ }_{n} \mathrm{~L}(F)$ to be the smallest number $d$ such that every element of $\mathrm{H}^{2}\left(F, \mu_{n}\right)$ can be written as a sum of $d \mathbb{Z} / n$-cyclic classes. See [1, Section 3] for a discussion of known results regarding $\mathbb{Z} / n$-length, usually called "symbol length" when $F$ contains an $n$-th root of unity.

If ${ }_{n} \mathrm{~L}(F)=d$ then it is clear that ${ }_{n} \operatorname{Br} \cdot \operatorname{dim}(F) \leq d$. Conversely, it is not known whether a finite Brauer dimension implies a finite $\mathbb{Z} / n$-length, or even that $\mathrm{H}^{2}\left(F, \mu_{n}\right)$ is generated by $\mathbb{Z} / n$-cyclic classes. However, when $n=\ell$ Merkurjev proved that $\mathrm{H}^{2}\left(F, \mu_{\ell}\right)$ is generated by classes of index $\ell$ ([6, Theorem 2]), hence for the fields considered in this paper $\mathrm{H}^{2}\left(F, \mu_{\ell}\right)$ is generated by $\mathbb{Z} / \ell$-cyclic classes by Saltman's cyclicity result.

2.2. General Conventions. Let $S$ be an excellent scheme and suppose $n$ is invertible on $S$. We write $\mathbb{Z} / n(r)$ for the étale sheaf $\mathbb{Z} / n$ twisted by an integer $r$, and $\mathrm{H}^{q}(S, r)=\mathrm{H}^{q}(S, \mathbb{Z} / n(r))$ for the étale cohomology group. If $S=\operatorname{Spec} A$ for a ring $A$, we write $\mathrm{H}^{q}(A, r)$. If $T$ is a subscheme of $S$ we write $\kappa(T)$ for its ring of meromorphic functions, which is the localization of $\mathrm{O}_{T}$ at its associated points. If $T \rightarrow S$ is a morphism of schemes then the restriction $\operatorname{res}_{S \mid T}: \mathrm{H}^{q}(S, r) \rightarrow \mathrm{H}^{q}(T, r)$ is defined, and we write $\beta_{T}=\operatorname{res}_{S \mid T}(\beta)$, and if $T=\operatorname{Spec} B$ we write $\beta_{B}$. If $Z \subset S$ is another morphism we write $Z_{T}$ for the fiber product $Z \times{ }_{S} T$.

If $v$ is a valuation on a field $F$, we write $\kappa(v)$ for the residue field of the valuation ring $\mathrm{O}_{v}$, and $F_{v}$ for the completion of $F$ with respect to $v$. If $v$ arises from a prime divisor $D$ on $S$, we write $v=v_{D}, \kappa(D)$, and $F_{D}$. If a set $\left\{v_{i}\right\}$ arises from a divisor $D=\sum_{i} D_{i}$, we write $F_{D}=\prod_{i} F_{D_{i}}$. Recall that if $F=(F, v)$ is a discretely valued field and $\alpha \in \mathrm{H}^{q}(F, r)$, then $\alpha$ has a residue $\partial_{v}(\alpha)$ in $\mathrm{H}^{q-1}(\kappa(v), r-1)$. More generally if $\xi$ is a generic point of a scheme $S, F=\kappa(\xi)$, and $\alpha \in \mathrm{H}^{q}(S, r)$, then for 
each discrete valuation $v$ on $F$ we define

$$
\partial_{v}(\alpha) \stackrel{\mathrm{df}}{=} \partial_{v}\left(\alpha_{F}\right) \in \mathrm{H}^{q-1}(\kappa(v), r-1)
$$

We say $\alpha$ is unramified with respect to $v$ if $\partial_{v}(\alpha)=0$, and in that case the value of $\alpha$ at $v$ is the element $\alpha(v)=\operatorname{res}_{F \mid F_{v}}(\alpha) \in \mathrm{H}^{q}(\kappa(v), r) \leq \mathrm{H}^{q}\left(F_{v}, r\right)$ ([4, 7.13, p.19]). If $v$ arises from a prime divisor $D$ on a scheme, we will substitute the notations $\partial_{D}$ and $\alpha(D)$. If $S$ is noetherian we write $D_{\alpha}$ for the ramification divisor of $\alpha$ on $S$, which is the sum of (finitely many) prime divisors on $S$ at which $\alpha$ ramifies.

2.3. Setup. In the following, $F$ will always be a finitely generated field extension of $\mathbb{Q}_{p}$ of transcendence degree one, $n$ will be a prime-to- $p$ number, and $X / \mathbb{Z}_{p}$ will be a connected regular (projective, flat) relative curve over $\mathbb{Z}_{p}$ with function field $F=K(X)$. Such a surface exists for any $F$ by a theorem of Lipman (see [5, Theorem 8.3.44]). We write $X_{0}=X \otimes_{\mathbb{Z}_{p}} \mathbb{F}_{p}$ for the closed fiber, $C=X_{0 \text {,red }}$ for the reduced scheme underlying the closed fiber, $C_{1}, \ldots, C_{m}$ for the irreducible components of $C$, and $\mathcal{S}$ for the set of singular points of $C$. We assume that $X_{0}$ has normal crossings, hence that each $C_{i}$ is regular, and at most two of them meet (transversally) at each singular point of $C$. This is situation is always achievable, by embedded resolution of curves in surfaces (see [5, Theorem 9.2.26]).

We say an effective divisor $D$ on $X$ is horizontal if each of its irreducible components maps surjectively to Spec $\mathbb{Z}_{p}$. By [3, Proposition 2.6] there exists for each closed point $z \in X \backslash \mathcal{S}$ a regular irreducible horizontal divisor $D \subset X$ that intersects $C$ transversally. Let $\mathscr{D}_{\mathcal{S}}$ denote the support of these lifts. We say a divisor $D$ is distinguished and write $D \in \mathscr{D}_{\mathcal{S}}$ if it is reduced and supported in $\mathscr{D}_{\mathcal{S}}$. Each $D \in \mathscr{D}_{\mathcal{S}}$ is a disjoint union of its irreducible components, each of which has a single closed point and meets $C$ transversally.

By weak approximation ([9, Lemma]) there exists an element $\pi \in F$ such that $\operatorname{div}(\pi)=C+E \subset X$, where $E$ is horizontal and avoids all closed points of any finite set. We fix such a $\pi$ such that $\operatorname{div}(\pi)$ avoids $\mathcal{S}$.

Suppose $\ell \neq p$ is prime, $\alpha \in \mathrm{H}^{2}\left(F, \mu_{\ell}\right)$, and $D_{\alpha} \subset X$ has normal crossings. Following Saltman's terminology in [11] we say $\alpha$ has a hot point $z$ on $X$ if (and only if) $z$ is a nodal point of $D_{\alpha}$, and if $D, D^{\prime} \subset D_{\alpha}$ are the two irreducible components meeting transversally at $z$, then $\partial_{D}(\alpha)$ and $\partial_{D^{\prime}}(\alpha)$ are unramified at $z$, and $\left\langle\partial_{D}(\alpha)(z)\right\rangle \neq\left\langle\partial_{D^{\prime}}(\alpha)(z)\right\rangle$. By [11, Corollary 5.2], $\alpha$ has index $\ell$ if and only if $D_{\alpha}$ has no hot points (hot point criterion), and by [11, Theorem 5.1], if $\alpha$ has index $\ell$ then it is cyclic.

Theorem 2.4 ([3, Lemma 4.6, Theorem 4.9]). Assume the setup of (2.3).

a) The image of the natural map $\mathrm{H}^{1}\left(\mathrm{O}_{C, \mathcal{S}}, \mathbb{Z} / n\right) \rightarrow \mathrm{H}^{1}(\kappa(C), \mathbb{Z} / n)$ consists of the set of tuples $\left(\theta_{1}, \ldots, \theta_{m}\right) \in \mathrm{H}^{1}(\kappa(C), \mathbb{Z} / n)$ such that each $\theta_{i}$ is unramified at each $z \in \mathcal{S} \cap C_{i}$, and $\theta_{i}(z)=\theta_{j}(z) \in \mathrm{H}^{1}(\kappa(z), \mathbb{Z} / n)$ whenever $z \in C_{i} \cap C_{j}$.

b) For $q \geq 0$ and any integer $r$ there is a map

$$
\lambda: \mathrm{H}^{q}\left(\mathrm{O}_{C, \mathcal{S}}, r\right) \rightarrow \mathrm{H}^{q}(F, r)
$$


and a commutative diagram

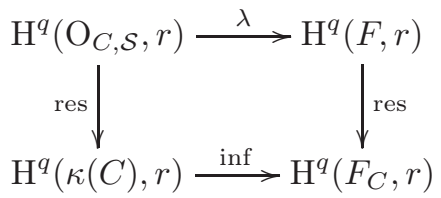

such that if $\alpha_{C} \in \mathrm{H}^{q}\left(\mathrm{O}_{C, \mathcal{S}}, r\right)$ and $\alpha=\lambda\left(\alpha_{C}\right)$ then:

i) $\alpha$ is defined at the generic points of $C_{i}$, and $\alpha\left(C_{i}\right)=\operatorname{res}_{\mathrm{O}_{C, \mathcal{S}}} \mid \kappa\left(C_{i}\right)\left(\alpha_{C}\right)$.

ii) The ramification locus of $\alpha($ on $X)$ is contained in $\mathscr{D}_{\mathcal{S}}$.

iii) If $D \in \mathscr{D}_{\mathcal{S}}$ is prime and $z=D \cap C$, then $\partial_{D} \cdot \lambda=\inf _{\kappa(z) \mid \kappa(D)} \cdot \partial_{z}$.

iv) If $\alpha_{C}$ is unramified at a closed point $z$, and $D$ is any (horizontal) prime lying over $z$, then $\alpha$ is unramified at $D$, and has value $\alpha(D)=\inf _{\kappa(z) \mid \kappa(D)}\left(\alpha_{C}(z)\right)$.

\section{Computations}

We first construct the cyclic class $\gamma \in \mathrm{H}^{2}\left(F, \mu_{n}\right)$ using a lift from $\mathrm{H}^{1}(\kappa(C), \mathbb{Z} / n)$.

Lemma 3.1. Assume the setup of (2.3). Suppose $\theta_{C} \in \mathrm{H}^{1}\left(\mathrm{O}_{C, \mathcal{S}}, \mathbb{Z} / n\right)$ maps to $\left(\theta_{1}, \ldots, \theta_{m}\right) \in \mathrm{H}^{1}(\kappa(C), \mathbb{Z} / n)$ as in Theorem 2.4 (a), such that $\theta_{C}$ is unramified at all $z \in E \cap C$, with value $\theta_{C}(z)=0$ (in addition to being unramified at $\mathcal{S}$ ). Let $\gamma=(\pi) \cdot \lambda\left(\theta_{C}\right) \in \mathrm{H}^{2}\left(F, \mu_{n}\right)$. Then for a prime divisor $D$ on $X$

$$
\partial_{D}(\gamma)= \begin{cases}\theta_{i} & \text { if } D=C_{i} \\ -\inf _{\kappa(z) \mid \kappa(D)}\left(\partial_{z}\left(\theta_{C}\right)\right) \cdot(\pi) & \text { if } D \in \mathscr{D}_{\mathcal{S}}, z=D \cap C, \text { and } v_{D}(\pi)=0 . \\ 0 & \text { otherwise }\end{cases}
$$

The ramification divisor $D_{\gamma}$ has normal crossings, and consists of each $C_{i}$ at which $\theta_{i}$ is nonzero, together with all $D \in \mathscr{D}_{\mathcal{S}}$ lifting $z: \partial_{z}\left(\theta_{C}\right) \neq 0$.

Proof. Set $\theta=\lambda\left(\theta_{C}\right)$. Let $D \subset X$ be a prime divisor and let $z=D \cap C$. Then

$$
\partial_{D}(\gamma)=\left[v_{D}(\pi) \theta-\partial_{D}(\theta) \cdot(\pi)+v_{D}(\pi) \partial_{D}(\theta) \cdot(-1)\right]_{F_{D}}
$$

This element is in the subgroup $\mathrm{H}^{1}(\kappa(D), \mathbb{Z} / n) \leq \mathrm{H}^{1}\left(F_{D}, \mathbb{Z} / n\right)$.

If $D=C_{i}$ is an irreducible component of $C$ then since $E$ contains no components of $C$ (by (2.3) ) we have $v_{D}(\pi)=1$, and since $\theta_{C} \in \mathrm{H}^{1}\left(\mathrm{O}_{C, \mathcal{S}}, \mathbb{Z} / n\right)$ we have $\partial_{D}(\theta)=0$ by Theorem 2.4(b)(ii). Therefore $\partial_{D}(\gamma)=\operatorname{res}_{F \mid F_{C_{i}}}(\theta)=\theta_{i} \in \mathrm{H}^{1}\left(\kappa\left(C_{i}\right), \mathbb{Z} / n\right)$. If $D \in \mathscr{D}_{\mathcal{S}}$ and $v_{D}(\pi)=0$ then $\partial_{D}(\gamma)=-\partial_{D}(\theta) \cdot(\pi)=-\inf _{\kappa(z) \mid \kappa(D)}\left(\partial_{z}\left(\theta_{C}\right)\right) \cdot(\pi)$ by Theorem 2.4(b)(iii).

It remains to show $\partial_{D}(\gamma)=0$ if $D$ is horizontal and runs through a point of $\mathcal{S}$, if $D$ is horizontal and $v_{D}(\pi) \neq 0$, or if $D \notin \mathscr{D}_{\mathcal{S}}$. If $D$ is horizontal and runs through a point of $\mathcal{S}$, then $v_{D}(\pi)=0$ since $E$ avoids $\mathcal{S}$, and $\partial_{D}(\theta)=0$ by Theorem 2.4(b)(ii), hence $\partial_{D}(\gamma)=0$. If $D$ is horizontal, avoids $\mathcal{S}$, and $v_{D}(\pi) \neq 0$, then $D$ is a component of $E$, so by assumption, $\partial_{z}\left(\theta_{C}\right)=0$ for $z \in E \cap C$ and $\theta_{C}(z)=0$. Thus $\partial_{D}(\theta)=0$ and $\theta(D)=0$ by Theorem 2.4(b)(iv). Therefore $\partial_{D}(\gamma)=0$. Finally, if $D$ is horizontal, avoids $\mathcal{S}, v_{D}(\pi)=0$, and $D \notin \mathscr{D}_{\mathcal{S}}$ then $\partial_{D}(\gamma)=-\partial_{D}(\theta) \cdot(\pi)=0$ since $\partial_{D}(\theta)=0$ by Theorem 2.4(b)(ii). 
Suppose $D \in \mathscr{D}_{\mathcal{S}}$ and $v_{D}(\pi)=0$, so $\partial_{D}(\gamma)=-\inf _{\kappa(z) \mid \kappa(D)}\left(\partial_{z}\left(\theta_{C}\right)\right) \cdot(\pi)$, where $z=D \cap C$. Since $v_{D}(\pi)=0, \pi$ is a local equation for $C$ at $z$ and $D$ intersects $C$ transversally at $z$, the image of $\pi$ in the local field $\kappa(D)$ is a uniformizer, hence $(\pi)$ has order $n$ in $\mathrm{H}^{1}\left(\kappa(D), \mu_{n}\right)$. Thus $\partial_{D}(\gamma)$ is nonzero in this case if and only if $\partial_{z}\left(\theta_{C}\right) \neq 0$.

We conclude $D_{\gamma}$ consists of the components $C_{i}$ of $C$ for which $\theta_{i}$ is nonzero, together with the distinguished prime divisors $D \in \mathscr{D}_{\mathcal{S}}$ lying over points $z$ at which $\theta_{C}$ is ramified. Since all such $D$ are regular and intersect $C$ transversally, $D_{\gamma}$ has normal crossings.

Next we show an $F$-division algebra of prime period $\ell \neq p$ and index $\ell^{2}$ is decomposable by constructing a cyclic factor using Lemma 3.1. designed to cancel the division algebra's hot points.

Theorem 3.2. Let $F$ be a field finitely generated of transcendence degree one over $\mathbb{Q}_{p}$, and suppose $\Delta$ is an $F$-division algebra of prime period $\ell \neq p$ and index $\ell^{2}$. Then $\Delta$ is decomposable.

Proof. We may assume $\ell$ is odd, since if $\ell=2$ the result is a classical theorem of Albert. Assume the setup (2.3), let $\alpha=[\Delta] \in \mathrm{H}^{2}\left(F, \mu_{\ell}\right)$, and let $D_{\alpha}$ be the ramification divisor of $\alpha$ on $X$. We may assume $D_{\alpha} \cup C$ has normal crossings and horizontal components contained in $\mathscr{D}_{\mathcal{S}}$, and that we have an element $\pi \in F$ as in (2.3) with $\operatorname{div}(\pi)=C+E$, where $E$ is horizontal and avoids the nodal points of $D_{\alpha} \cup C$. By Grunwald-Wang's theorem there exist elements $\theta_{i} \in \mathrm{H}^{1}\left(\kappa\left(C_{i}\right), \mathbb{Z} / \ell\right)$ such that

a) $\partial_{z}\left(\theta_{i}\right)=0$ when $z \in C_{i}$ is a singular point of $C \cup D_{\alpha} \cup E$.

b) $\theta_{i}(z)=\theta_{j}(z)$ whenever $z \in C_{i} \cap C_{j}$.

c) $\theta_{i}(z)=0$ at all $z \in E \cap C_{i}$.

d) If $z \in D_{\alpha} \cap \mathcal{S}$ then

(i) $\left\langle\partial_{C_{i}}(\alpha)(z)-\theta_{i}(z)\right\rangle=\left\langle-\theta_{i}(z)\right\rangle$ if $z \in C_{i}$ is a curve point of $D_{\alpha}$;

(ii) $\theta_{i}(z)=0$ if $z \in C_{i}$ is a not-hot nodal point of $D_{\alpha}$;

(iii) $\left\langle\partial_{C_{i}}(\alpha)(z)-\theta_{i}(z)\right\rangle=\left\langle\partial_{C_{j}}(\alpha)(z)-\theta_{i}(z)\right\rangle$ if $z \in C_{i} \cap C_{j}$ is a hot point of $\alpha$.

e) If $z \in D_{\alpha} \backslash \mathcal{S}$ then

(i) $\left\langle-\theta_{i}(z)\right\rangle=\left\langle\partial_{D}(\alpha)(z)\right\rangle$ if $z \in C_{i}$ is a curve point of $D_{\alpha}$;

(ii) $\theta_{i}(z)=0$ if $z \in C_{i}$ is a not-hot nodal point of $D_{\alpha}$;

(iii) $\left\langle\partial_{C_{i}}(\alpha)(z)-\theta_{i}(z)\right\rangle=\left\langle\partial_{D}(\alpha)(z)\right\rangle$ if $z \in C_{i} \cap D$ is a hot point of $\alpha$.

Note that we may arrange (a), (b), and (c) since the given point sets are finite; (d)(i,iii) and (e)(i,iii) makes sense since the given residues of $\alpha$ are unramified at the given $z ;(\mathrm{d})(\mathrm{i}, \mathrm{iii})$ and (e)(iii) are possible since $\ell$ is odd; (c) does not conflict with (d)(i,iii) and (e)(i,iii) since $E$ avoids the nodal points of $D_{\alpha} \cup C$; and (b) does not conflict with (d)(ii,iii) and (e)(ii,iii) by symmetry.

The $\theta_{i}$ are unramified with equal values at all nodal points $z \in \mathcal{S}$ by $(\mathrm{a}, \mathrm{b})$, so there exists an element $\theta_{C} \in \mathrm{H}^{1}\left(\mathrm{O}_{C, \mathcal{S}}, \mathbb{Z} / \ell\right)$ mapping to $\left(\theta_{1}, \ldots, \theta_{m}\right) \in \mathrm{H}^{1}(\kappa(C), \mathbb{Z} / \ell)$ by Theorem 2.4(a). Note that $\theta_{C}$ is nonzero by (d)(iii) (or (e)(iii)) since $\alpha$ has at least one hot point by the hot point criterion [11, Corollary 5.2], and then $\theta_{C}(z)=\theta_{i}(z)$ is necessarily nonzero. 
Let $\gamma_{1}=(\pi) \cdot \lambda\left(\theta_{C}\right)$. Then $\gamma_{1}$ and $E$ satisfy the hypotheses of Lemma 3.1 by (c) and the assumptions on $E$, hence $D_{\gamma_{1}} \cup C$ has normal crossings and distinguished horizontal components, and since $\theta_{C}$ is ramified at all nodal points of $D_{\gamma_{1}}, \gamma_{1}$ has no hot points, hence it has index $\ell$ by the hot point criterion. Write

$$
\begin{aligned}
D_{\alpha} & =C^{\prime}+H \\
D_{\gamma_{1}} & =C^{\prime \prime}+H^{\prime}
\end{aligned}
$$

where $C^{\prime}, C^{\prime \prime} \subset C$, and $H, H^{\prime} \subset \mathscr{D}_{\mathcal{S}}$ are distinguished horizontal divisors. Set

$$
\gamma_{2}=\alpha-\gamma_{1}
$$

We intend to show that $\gamma_{2}$ has index $\ell$. Since $\theta_{C}$ is unramified at all singular points of $D_{\alpha} \cup C$ by (a), $H^{\prime}$ avoids all of these points by Lemma 3.1, hence $H \cap H^{\prime}=\varnothing$. Evidently $D_{\gamma_{2}} \subset C+H+H^{\prime}$, hence $D_{\gamma_{2}}$ has normal crossings on $X$. Since $D_{\gamma_{2}}$ has normal crossings, $\gamma_{2}$ has index $\ell$ if and only if $\gamma_{2}$ has no hot points on $X$.

For the following analysis, note the nodal points $\mathcal{S}_{\gamma_{2}}$ of $D_{\gamma_{2}}$ are in $H^{\prime}, \mathcal{S}$, and $H$, and in the latter two cases $\theta_{C}$ is unramified at $z$, hence has a value $\theta_{C}(z)$.

Suppose $z \in \mathcal{S}_{\gamma_{2}}$ and $z \notin D_{\alpha}$. Then $z$ 's status as a point of $D_{\gamma_{2}}$ (hot, not hot) is the same as its status as a point of $D_{\gamma_{1}}$, which is not hot since $\gamma_{1}$ has no hot points.

Suppose $z \in \mathcal{S}_{\gamma_{2}} \cap D_{\alpha} \cap H^{\prime}$. Then $z \in C_{i} \cap D$ for some $C_{i}$ and some prime divisor $D \subset H^{\prime}$, and $\theta_{C}$ is ramified at $z$ by Lemma 3.1. We have $\partial_{D}(\alpha)=0$ since $D \not \subset D_{\alpha}$, and by Lemma 3.1

$$
\partial_{z}\left(\partial_{D}\left(\gamma_{2}\right)\right)=\partial_{z}\left(-\partial_{D}\left(\gamma_{1}\right)\right)=\partial_{z}\left(\partial_{z}\left(\theta_{C}\right) \cdot(\pi)\right)=v_{z}(\bar{\pi}) \partial_{z}\left(\theta_{C}\right)
$$

where $\bar{\pi}$ is the image of $\pi$ in $\kappa(D)$. Since $\operatorname{div}(\pi)$ has normal crossings at $z, v_{z}(\bar{\pi})=1$, hence $\partial_{z}\left(\partial_{D}\left(\gamma_{2}\right)\right)=\partial_{z}\left(\theta_{C}\right) \neq 0$. Since $\partial_{z}\left(\partial_{D}\left(\gamma_{2}\right)\right) \neq 0, z$ is not a hot point of $\gamma_{2}$.

Suppose $z \in \mathcal{S}_{\gamma_{2}} \cap D_{\alpha} \cap \mathcal{S}$. If $z$ is a curve point of $D_{\alpha}$ on $C_{i}$, i.e., $\partial_{C_{j}}(\alpha)=0$ where $C_{j}$ is the other component of $C$ at $z$, then $\partial_{C_{i}}\left(\gamma_{2}\right)(z)=\partial_{C_{i}}(\alpha)(z)-\theta_{C}(z)$ and $\partial_{C_{j}}\left(\gamma_{2}\right)(z)=-\theta_{C}(z)$ by Lemma 3.1. and so $z$ is not a hot point of $\gamma_{2}$ by (d)(i). If $z$ is a nodal point of $D_{\alpha}$ on $C_{i} \cap C_{j}$, then $\theta_{C}(z)=0$ if $z$ is not a hot point of $\alpha$ by (d)(ii), so that the status of $z$ for $\gamma_{2}$ is the same as for $\alpha$ (not hot); otherwise $\left\langle\partial_{C_{i}}\left(\gamma_{2}\right)(z)\right\rangle=\left\langle\partial_{C_{j}}\left(\gamma_{2}\right)(z)\right\rangle$ by (d)(iii), hence $z$ is not a hot point for $\gamma_{2}$ in any case.

Suppose $z \in \mathcal{S}_{\gamma_{2}} \cap D_{\alpha} \cap H$. Assume $z \in C_{i} \cap D$ for a prime divisor $D \subset H$. Then $\partial_{D}\left(\gamma_{1}\right)=0$ since $H \cap H^{\prime}=\varnothing$. If $z$ is a curve point of $D_{\alpha}$, i.e., $\partial_{C_{i}}(\alpha)=0$, then $\partial_{C_{i}}\left(\gamma_{2}\right)(z)=-\theta_{C}(z)$ and $\partial_{D}\left(\gamma_{2}\right)=\partial_{D}(\alpha)$, hence $\left\langle\partial_{C_{i}}\left(\gamma_{2}\right)(z)\right\rangle=\left\langle\partial_{D}\left(\gamma_{2}\right)(z)\right\rangle$ by (e)(i), so $z$ is not a hot point for $\gamma_{2}$. If $z$ is a not-hot nodal point of $D_{\alpha}$ then $\theta_{C}(z)=0$ by (e)(ii), so the status of $z$ is unchanged (not hot) for $\gamma_{2}$. If $z$ is a hot point of $\alpha$ then $\left\langle\partial_{C_{i}}(\alpha)(z)-\theta_{C}(z)\right\rangle=\left\langle\partial_{D}(\alpha)(z)\right\rangle$ by (e)(iii), hence $z$ is not a hot point for $\gamma_{2}$. This completes the analysis. We conclude $\gamma_{2}$ has no hot points on $X$, hence $\gamma_{1}$ and $\gamma_{2}$ both have index $\ell$.

Let $\Delta_{1}$ and $\Delta_{2}$ be the $F$-division algebras underlying $\gamma_{1}$ and $\gamma_{2}$, respectively, so that $[\Delta]=\left[\Delta_{1} \otimes_{F} \Delta_{2}\right]$. Since $\operatorname{ind}\left(\Delta_{1} \otimes_{F} \Delta_{2}\right)=\operatorname{ind}(\Delta)=\ell^{2}$ and $\operatorname{ind}\left(\Delta_{i}\right)=\ell$, it follows that $\Delta_{1} \otimes_{F} \Delta_{2}$ is a division algebra, hence $\Delta \simeq \Delta_{1} \otimes_{F} \Delta_{2}$.

As mentioned in (2.1), it is known that $\ell_{\ell} \operatorname{Br} \cdot \operatorname{dim}(F)=2$ and that the $\mathbb{Z} / \ell$-length ${ }_{\ell} \mathrm{L}(F)$ is finite. We now have the following. 
Corollary 3.3. Let $F$ be a field finitely generated and of transcendence degree one over $\mathbb{Q}_{p}$, and let $\ell \neq p$ be a prime. Then $\ell_{\ell} \mathrm{L}(F)=2$.

Proof. If $\alpha \in \mathrm{H}^{2}\left(F, \mu_{\ell}\right)$ then the index of $\alpha$ is either $\ell$ or $\ell^{2}$ by [8, Theorem 3.4]. If it is $\ell$, then $\alpha$ is already $\mathbb{Z} / \ell$-cyclic by [11, Theorem 5.1]. If it is $\ell^{2}$ then $\alpha=\gamma_{1}+\gamma_{2}$ for classes $\gamma_{i}$ in $\mathrm{H}^{2}\left(F, \mu_{\ell}\right)$ of index $\ell$ by Theorem 3.2 These classes are again $\mathbb{Z} / \ell$-cyclic by Saltman's theorem, and the result follows.

Saltman proved that all $F$-division algebras of prime degree $\ell$ are cyclic crossed products in [11, and Suresh proved the prime period case when $F$ contains the $\ell$-th roots of unity in 12. We now have the prime period case in general:

Corollary 3.4. Let $F$ be a field finitely generated and of transcendence degree one over $\mathbb{Q}_{p}$, and let $\Delta$ be a division algebra of prime period $\ell \neq p$. Then $\Delta$ is a crossed product.

Proof. The index of $\Delta$ is either $\ell$ or $\ell^{2}$ by [8, Theorem 3.4]. If it is $\ell$, then $\Delta$ is a cyclic crossed product by [11, Theorem 5.1]. If it is $\ell^{2}$ then $\Delta=\Delta_{1} \otimes_{F} \Delta_{2}$ by Theorem 3.2. and each $\Delta_{i}$ is cyclic by Saltman's theorem. Let $L_{i} / F$ be a cyclic Galois maximal subfield of $\Delta_{i}$. Then $L=L_{1} \otimes_{F} L_{2}$ is a commutative Galois subalgebra of $\Delta$ of degree $\ell^{2}$. Since $L$ obviously splits $\Delta, \Delta$ is a crossed product by 10, Theorem 7.2].

\section{REFERENCES}

[1] A. Auel, E. Brussel, S. Garibaldi, and U. Vishne. Open problems on central simple algebras. Transform. Groups, 16(1):219-264, March 2011.

[2] E. Brussel, K. McKinnie, and E. Tengan. Indecomposable and noncrossed product division algebras over function fields of smooth p-adic curves. Adv. in Math., 226:4316-4337, 2011.

[3] E. Brussel and E. Tengan. Tame covers and cohomology of relative curves over complete discrete valuation rings, with applications to the Brauer group. http://arxiv.org/abs/1104.0439, 2011.

[4] S. Garibaldi, A. Merkurjev, and J.-P. Serre. Cohomological invariants in Galois cohomology, volume 28 of University Lecture Series. Amer. Math. Soc., 2003.

[5] Q. Liu. Algebraic Geometry and Arithmetic Curves, volume 6 of Oxford Graduate Texts in Mathematics. Oxford University Press, Oxford, 2002. Translated from the French by Reinie Erné, Oxford Science Publications.

[6] A. Merkurjev. Brauer groups of fields. Comm. Alg., 11:2611-2624, 1983.

[7] A. Merkurjev and A. Suslin. K-cohomology of Severi-Brauer varieties and the norm residue homomorphism. Math. USSR Izv., 21(2):307-340, 1983.

[8] D. Saltman. Division algebras over p-adic curves. J. Ramanujan Math. Soc., 12:25-47, 1997. see also the erratum [9] and survey [?].

[9] D. Saltman. Correction to division algebras over p-adic curves. J. Ramanujan Math. Soc., 13:125-129, 1998.

[10] D. Saltman. Lectures on Division Algebras, volume 94 of CBMS Regional Conference Series in Mathematics. American Mathematical Society, Providence, RI, 1999.

[11] D. Saltman. Cyclic algebras over p-adic curves. J. Algebra, 314:817-843, 2007.

[12] V. Suresh. Bounding the symbol length in the Galois cohomology of function fields of $p$-adic curves. Comment. Math. Helv., 85(2):337-346, 2010.

Department of Mathematics \& Computer Science, Emory University, Atranta, Ga 30322, USA

E-mail address: brussel@mathcs.emory.edu 
Instituto de Ciências Matemáticas e de Computação, Universidade de São Paulo, São Carlos, São Paulo, Brazil

E-mail address: etengan@icmc.usp.br 